\title{
Un enjeu politique pour le gouvernement britannique
}

Apprendre à lire et à écrire

A political issue for the British government. Teaching reading and writing

Una apuesta politica para el gobierno británico. Aprender a leer y a escribir

\section{Carole King}

Traducteur : Jean-Paul Gratias

\section{OpenEdition}

\section{Journals}

Édition électronique

URL : http://journals.openedition.org/ries/2792

DOI : $10.4000 /$ ries. 2792

ISSN : 2261-4265

Éditeur

Centre international d'études pédagogiques

Édition imprimée

Date de publication : 1 mars 1999

Pagination : 53-63

ISBN : 9771254459005

ISSN : 1254-4590

\section{Référence électronique}

Carole King, "Un enjeu politique pour le gouvernement britannique », Revue internationale d'éducation de Sèvres [En ligne], 21 | mars 1999, mis en ligne le 16 avril 2013, consulté le 08 janvier 2020. URL: http://journals.openedition.org/ries/2792 ; DOI : 10.4000/ries.2792

Ce document a été généré automatiquement le 8 janvier 2020

(c) Tous droits réservés 


\title{
Un enjeu politique pour le gouvernement britannique
}

\author{
Apprendre à lire et à écrire \\ A political issue for the British government. Teaching reading and writing \\ Una apuesta politica para el gobierno británico. Aprender a leer y a escribir
}

\section{Carole King}

Traduction : Jean-Paul Gratias

« Tous nos enfants méritent de quitter l'école en possession des outils qui leur permettront d'aborder une vie d'adulte dans laquelle ils pourront s'épanouir. Mais si les enfants ne maîtrisent pas les savoir-faire élémentaires de la lecture, de l'écriture et du calcul quand ils sont à l'école primaire, ils seront gravement handicapés par la suite ${ }^{1} »$

\section{Ellis et Tilda sont dans un bateau..}

1 Rentrant récemment en bateau d'une excursion d'une journée à Dieppe, je me suis retrouvée entourée de familles ayant de jeunes enfants. À ma gauche se trouvait Ellis, dont la mère garda le silence pendant tout le voyage ; elle n'adressait la parole à son fils que pour le réprimander, critiquant tout ce qu'il faisait, tout ce qu'il disait. C'est la grand-mère qui faisait la conversation à l'enfant, en profitant pour lui glisser un couplet sur la nécessité de bien travailler à l'école dès son retour : «Car, dit-elle, tu vas avoir sept ans cette année et tu ne sais pas encore lire ni écrire ».

2 Ellis, qui boudait parce que l'entrée de la salle des jeux électroniques lui était interdite, n'avait d'autre distraction que l'écran vidéo qui diffusait au-dessus de sa tête un dessin animé muet. À ma droite était assise Tilda, qui, à quatre ans et demi, lisait couramment et prenait plaisir à écrire dans son petit journal de bord et à faire des jeux de lettres. 
Son père et sa mère dévoraient chacun un livre, mais prenaient le temps de lui faire la lecture, ainsi qu'à sa petite sœur. Et quand Tilda, brièvement, leva les yeux vers l'écran vidéo, elle reconnut l'histoire de Rapunzel et commença à parler de la version qu'elle possédait chez elle. Deux enfants dont la conception de la lecture et le cheminement vers l'acquisition de ce savoir-faire semblent très différents, deux enfants qui, de façon différente, représentent un défi à relever pour leurs enseignants. «Maîtriser les savoirfaire élémentaires » est sans doute vital pour réussir une vie d'adulte, mais tandis que la grand-mère d'Ellis, manifestement, considérait que l'apprentissage de la lecture et de l'écriture était l'affaire du maitre d'école, les parents de Tilda n'y voyaient qu'une facette supplémentaire de l'éducation qu'il était de leur devoir de donner à leur enfant - non pas un " plus » fourni par une structure extérieure, mais une évolution naturelle encouragée à la maison et développée par l'école.

\section{Réformes, changements, révisions}

3 En Angleterre, depuis le mois de septembre 1998, les écoles primaires ${ }^{2}$ (primary schools) et les institutions ${ }^{3}$ assurant la formation initiale des enseignants sont tenues de mettre en œuvre des modifications importantes du programme. Ces changements ont pour objectif d'assurer aux élèves une meilleure maitrise de la lecture et de l'expression écrite, principalement pour préparer de futurs adultes plus compétitifs sur le marché du travail. Ils reflètent non seulement le contrôle centralisé, de plus en plus pressant, que fait peser le ministère sur les salles de cours aussi bien des écoles que des universités, mais aussi sa tendance grandissante, dans sa quête de solutions simples à des problèmes anciens, à ignorer ce qu'ont de complexes la maîtrise de la lecture et de l'écriture ainsi que les savoir-faire concernés. Quelle est la meilleure façon d'apprendre à des enfants à lire et à écrire, et comment devons-nous former nos enseignants à s'acquitter efficacement de cette tâche? Le professionnalisme et l'autonomie des enseignants de tous niveaux courent-ils le risque d'être annihilés par les forces conservatrices de la droite qui ne croient pas les enseignants capables de faire le travail eux-mêmes?

Cela fait déjà dix ans que les écoles subissent une période de changements intensifs. Cela a commencé avec l'introduction du National Curriculum qui déterminait à la fois la nature et le contenu des matières enseignées, et la création d'un corps d'inspection ${ }^{4}$ chargé de veiller à son application. Le curriculum officiel pour l'enseignement de l'anglais, l'un des premiers a être mis au point par le professeur Cox et son équipe en 1989, fut généralement bien accueilli par les institutions de formation, car il s'appuyait sur une connaissance et une intelligence approfondies de l'enseignement et de l'apprentissage de la lecture et de l'écriture, reflétant la recommandation de Bruner ${ }^{5}$ selon laquelle «le programme d'enseignement d'une matière devrait être déterminé par la compréhension la plus fondamentale possible des principes sous-jacents qui structurent cette matière ». Ce curriculum reconnaissait aussi que l'anglais, quelle que soit son importance en tant que médium grâce auquel les autres matières sont enseignées, devait également prendre en compte les dimensions personnelle, sociale et culturelle de la maîtrise de la langue et de la littérature, tout en préparant les enfants à la vie adulte. Un autre axe majeur de ce programme était la nécessité de développer chez l'élève une analyse critique de la façon dont sont construits des textes de toutes sortes et dont ils produisent du sens. 
Cependant, moins de cinq ans plus tard (1993), et avant qu'il eût été appliqué à tous les niveaux du primaire, ce curriculum fut révisé parce qu'il «ne définissait pas avec une clarté suffisante les connaissances et les compétences essentielles que devait promouvoir l'enseignement de l'anglais, et ne fournissait donc pas la meilleure structure possible pour élever le niveau des élèves en expression et en compréhension orale, en lecture et en expression écrite $»^{6}$. La version révisée, fortement influencée par des personnalités nettement situées à droite (appartenant ou non au monde de l'enseignement), penchait vers une conception plus utilitaire de l'anglais. En expression écrite, par exemple, alors que Cox avait constaté que «la langue écrite remplit de nombreuses fonctions aussi bien pour les individus que pour la société dans son

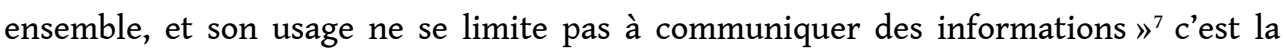
compétence communicative que l'on privilégie désormais. Aux premiers stades de l'apprentissage de la lecture, on accorde une place beaucoup plus importante à la description du travail à effectuer sur la prononciation et sur la graphie des mots. Ceci reflète l'influence des traditionalistes, pour qui apprendre à lire est un processus ascendant qui commence par l'apprentissage de sons isolés, plutôt qu'une démarche qui vise à construire du sens en abordant des textes entiers de différentes façons.

6 Pourtant, en dépit des modifications du curriculum et des évaluations nationales auxquelles sont soumis les élèves de sept, onze et quatorze ans, l'enseignement de la lecture est toujours considéré par le gouvernement comme un secteur problématique où les résultats obtenus demeurent insatisfaisants. Les résultats des recherches, cependant, tendent à prouver le contraire. Mais ce qui n'est pas contestable, c'est que l'Angleterre traîne derrière elle "une longue tradition d'échec scolaire " qui n'est guère flatteuse, comparée aux résultats des autres pays développés, une tradition à laquelle appartiennent assurément des enfants comme Ellis, alors que Tilda, quand elle aura onze ans, figurera très probablement parmi les $20 \%$ d'élèves dont le niveau dépasse déjà celui que l'on attend des enfants de cet âge ${ }^{8}$.

7 Pour s'attaquer à ce problème et pour élever le niveau général afin que, d'ici à 2002, $80 \%$ des élèves de 11 ans soient capables d'atteindre l'objectif fixé comme valeur médiane théorique, le gouvernement actuel a introduit des changements plus radicaux sous la forme d'une "stratégie nationale pour la lecture ${ }^{9}$ ». Il s'agit d'une initiative ambitieuse qui part du principe que la maîtrise des savoir-faire en lecture et en expression écrite ne peut être améliorée sans la participation de la communauté au sens le plus large. De fait, l'année scolaire 1998/1999 a été décrétée " Année nationale de la lecture ${ }^{10}$ » et les parents, les entreprises et les médias sont appelés à y apporter leur contribution. Au moment où j'écris ces lignes, l'émission de radio à grande audience Woman's Hour s'emploie à faire connaître l'Année nationale de la lecture, à expliquer aux parents quelle aide ils peuvent apporter et diffuse, par exemple, un entretien avec le Premier ministre : un père de famille qui fait la lecture à ses enfants. Il reste à voir si l'on atteindra, à la fin de l'année, l'objectif avoué qui est de «susciter l'intérêt de chaque citoyen et la participation de plusieurs millions d'entre eux ». Ce qui est certain, en revanche, c'est que les écoles tout comme les universités sont censées se conformer dès cette année aux nouvelles initiatives concernant l'enseignement de la lecture et de l'expression écrite. 


\section{La stratégie nationale pour la lecture}

8 Pour compléter le National Curriculum, le ministère a publié un plan de travail exhaustif qui recense en détail ce qui doit être enseigné, y compris les formes littéraires se prêtant à la lecture et à l'expression écrite pour chaque trimestre de chaque année scolaire du cycle primaire. Ce document, The National Literacy Strategy : a Framework for Teaching a été décrit par un de ses adeptes comme étant «la chair qui habille le squelette du National Curriculum ${ }^{11}$ ». Il est conçu pour garantir qu'un enseignement explicite de la lecture et de l'écriture soit donné quotidiennement dans toutes les classes du primaire, agencé selon trois axes principaux interconnectés :

- au niveau du mot, prononciation, graphie et lexique ;

- au niveau de la phrase, grammaire et ponctuation ;

- au niveau du texte, compréhension et composition.

9 Si l'on excepte les établissements qui ont choisi un statut autonome, les enseignants ont également l'obligation de dispenser ce contenu imposé dans un créneau connu sous le nom de Literacy Hour. Ils doivent consacrer une heure chaque jour à un enseignement en continu de la lecture et de l'expression écrite, et l'heure en question doit combiner des activités en classe entière et des activités de pédagogie différenciée. L'heure se termine par une mise en commun qui permet de revoir les points importants, d'inviter les élèves à une réflexion personnelle et de consolider les acquis. Une certaine flexibilité est inévitable dans le cadre de cette heure, mais les principes généraux sont censés être respectés. Les écoles doivent aussi utiliser les journées de formation et le matériel pédagogique fourni pour permettre à tous les enseignants de mettre en œuvre la même approche de la lecture et de l'expression écrite.

Bien qu'il y ait beaucoup d'aspects positifs, à la fois dans le modèle de référence et dans l'heure de travail structurée - en terme de stratégie pédagogique, dans le choix de textes véritables et dans l'idée de développer et d'expliciter la connaissance implicite que les enfants ont de la langue - aucune déclaration de principe n'a jusqu'à présent été fournie pour justifier cette approche, bien qu'il y en ait une, apparemment, en cours de rédaction. Nous sommes donc en présence d'un modèle de mise en œuvre du programme qui considère les maîtres comme des techniciens, censés enseigner d'une certaine façon sans comprendre pourquoi et, bien qu'on remarque chez eux un certain enthousiasme, on constate aussi beaucoup d'inquiétudes face aux exigences du modèle de référence. Les bons enseignants auront déjà parcouru, sans doute, une bonne partie $\mathrm{du}$ chemin prescrit, mais à leur manière et en tenant compte du profil de leurs propres élèves. Cependant, il est peu probable que les gens peu doués pour enseigner la lecture s'améliorent en se contentant d'appliquer les principes qu'on leur impose.

11 À ce stade, l'absence d'une déclaration de principe est inquiétante pour le professionnalisme des enseignants. Cependant, il en existe une concernant l'introduction du plan lui-même, puisque :

«Il n'y a jamais eu d'initiative nationale d'envergure pour permettre à tous les enseignants du primaire d'acquérir les méthodes les plus efficaces d'enseignement de la lecture et la façon de les appliquer. Le gouvernement précédent n'a pas mis en place les structures ni les incitations nécessaires pour faire en sorte que toutes les écoles tirent profit des meilleures pratiques pédagogiques des écoles les plus performantes. Au lieu de cela, il y a eu une série d'initiatives, valables mais isolées, qui n'ont eu ni l'envergure ni l'ambition nécessaire pour s'attaquer au problème sur l'ensemble du pays. Si les enseignants doivent changer, ils ont besoin qu'on leur 
donne la possibilité de découvrir les meilleures pratiques de classe et qu'on les incite à les adopter. À plus long terme, il nous faut une culture dans laquelle les maîtres du primaire eux-mêmes auront à cœur, pour satisfaire leur conscience professionnelle, d'adopter les meilleures méthodes ${ }^{12}$.»

12 Cependant aucun résultat de recherche pertinent n'est cité à l'appui de ces prétendues "meilleures approches», et bien que l'heure de lecture ait été depuis peu mise en œuvre, elle n'a pas encore été complètement évaluée. Toute initiative qui concentre les efforts de l'école entière sur l'acquisition de la lecture a des chances de produire, dans les premiers temps, quelques résultats. Le véritable test de validité de cette procédure consiste à savoir s'il s'agit de bénéfices durables et s'il est possible de maintenir la même structure tout au long des sept années du cycle primaire, et peut-être au-delà, lorsque le secondaire ${ }^{13}$ sera concerné. La diligence avec laquelle cette politique doit être mise en place reflète sans doute l'impatience du gouvernement à résorber l'échec scolaire, mais il est également possible que « les effets de la stratégie nationale pour la lecture doivent être évidents avant la prochaine élection ${ }^{14}$ ».

13 Étonnamment, bien que les formateurs en pédagogie aient un rôle majeur à jouer dans la mise en œuvre de cette initiative, ils ont été oubliés lors de sa présentation et, tandis que des stages étaient organisés à l'intention des chefs d'établissement, des représentants des conseils d'administration et des coordinateurs de langues vivantes, on laissa le soin aux établissements du supérieur de découvrir par leurs propres moyens de quoi il retournait. Il s'agit là d'une omission majeure, les universités préparant alors leurs contingents d'étudiants à obtenir leur certificat final(QTS) l'année même de l'entrée en vigueur de la stratégie nationale pour la lecture, omission d'autant plus surprenante que, selon l'opinion générale, il est primordial que les écoles et les universités accordent leurs violons, afin que «les pratiques pédagogiques en classe renforcent ce qui a été appris dans les instituts de formation ${ }^{15} »$.

\section{L'anglais dans le primaire : un enjeu politique?}

14 Cependant, si les instituts de formation ont été en grande partie laissés dans l'ignorance en ce qui concerne l'heure de lecture elle-même, il est évident que le nouveau Initial Teacher Training National Curriculum for Primary English a été conçu pour les préparer à l'enseigner. Son introduction est la dernière d'une série de mesures prises par la Teacher Training Agency (TTA), nouvellement créée, un organisme responsable du recrutement et de la formation de tous les enseignants du service public et de l'inspection de toutes les formations pédagogiques initiales. Le document, sous forme de projet, a pu être consulté dès 1996 et, à partir de septembre 1998, toutes les universités ont dû mettre en place la version définitive. Comme la stratégie nationale pour la lecture, il complète les textes précédents, considérés comme trop vagues pour être efficaces :

«La formation des maîtres du primaire est actuellement régie par la circulaire 14/93 qui décrit en détail les exigences du cursus et dresse une longue liste des compétences que les futurs enseignants sont censés acquérir avant la fin de leur formation. Les défauts de cette circulaire résident dans le fait qu'elle est trop imprécise et ne dégage pas de priorités suffisamment nettes ${ }^{16}$. $»$

15 Ainsi, les universités comme les écoles doivent enseigner comme on leur dit de le faire et se plier aux exigences des politiques qui les gouvernent et qui publient anonymement les instructions officielles. Ce coup porté à la liberté de l'université est 
aggravé par le fait que, si les universités ne parviennent pas à satisfaire à ces exigences, elles verront probablement fondre les effectifs de leurs étudiants. Concrètement, elles doivent former leurs étudiants à apprendre aux enfants à lire et à écrire dans le cadre de l'heure de lecture, stratégie dont l'efficacité reste à démontrer.

Le Training Curriculum, long de vingt pages, est divisé en trois chapitres :

- les connaissances et les facultés d'analyse requises chez les élèves-professeurs pour assurer les progrès des élèves en anglais (chapitre A) ;

- les méthodes efficaces d'enseignement et d'évaluation (chapitre B);

- la connaissance et la compréhension de l'anglais chez les élèves professeurs (chapitre C).

17 La partie du programme concernant l'expression écrite suggère en elle-même un modèle de maîtrise de l'écrit qui s'éloigne de celle du National Curriculum. Elle insiste encore plus sur la langue utilisée à des fins de communication plutôt que pour construire du sens. Pour chaque aspect, on distingue les «connaissances et facultés d'analyse requises chez tous les élèves-professeurs" et "la pertinence de ces connaissances par rapport à l'enseignement primaire» et ceci fait l'objet d'une évaluation tout au long de la formation, pour vérifier que les futurs enseignants possèdent bien les dites compétences. Mais il existe à ce propos un grave danger : s'il ne comprennent pas réellement les processus de lecture et d'expression écrite, les enseignants se concentrent sur les compétences purement techniques, par exemple la capacité d'un élève à identifier des propositions subordonnées sans reconnaître leur contribution au sens du texte, en restant à la surface du texte plutôt qu'en explorant les structures profondes de la langue, niant par là même une bonne partie de ce qu'on a appris depuis une quarantaine d'années sur la langue, les processus mentaux et la compréhension de l'écrit.

18 Alors qu'une bonne partie des recommandations de ce programme sont déjà incluses, ou peuvent l'être aisément, dans notre formation pédagogique, nous devons continuer à offrir à nos étudiants une conception plus large de la maîtrise de la lecture et de l'expression écrite. De plus en plus, aujourd'hui, cette conception doit inclure la façon dont l'informatique modifie nos critères de référence, pour aller bien au-delà de la définition simple donnée par le secrétaire d'État qui se contente de "savoir lire et écrire ${ }^{17}$.

Étant donné les contraintes très strictes des horaires de formation, les élèvesprofesseurs qui suivent un cursus en quatre ans et les licenciés préparant leur certificat professionnel (QTS) en un an ont un énorme travail à fournir s'ils veulent atteindre le niveau de connaissances requis par les programmes officiels, d'autant plus que les sciences, les mathématiques et l'informatique sont affectés des mêmes coefficients à l'examen final. Les connaissances théoriques telles que celles définies dans la section C sont importantes, mais elles ne garantissent pas à elles seules un enseignement efficace de la lecture et de l'expression écrite, ainsi que l'a montré l'enquête, non publiée, menée sur ce sujet par la TTA. En fait, il semble plus probable que les connaissances théoriques des enseignants soient pédagogiquement intégrées, acquises en relation directe avec la transmission de savoir et non pas séparément. Pour une pédagogie efficace, plutôt que d'être capables de détecter des phonèmes isolés ou des propositions subordonnées, il semble plus important encore que les enseignants comprennent les processus fondamentaux impliqués dans l'enseignement et l'apprentissage de la lecture et de l'expression écrite ${ }^{18}$. 


\section{Les universités confrontées à un défi} étudiants les expériences les plus riches possibles dans le domaine de la lecture, de l'expression écrite et de leur enseignement, tout en nous conformant, dans le même temps, aux exigences des textes officiels. Comme les écoles, nous sommes constamment sous la menace d'une visite du corps d'inspection. Au cours des deux dernières années, toutes les universités proposant une préparation en un an au diplôme de certification (QTS) ont été inspectées, de façon plutôt vigoureuse, pour leur enseignement de la lecture et du calcul. Elles ont ensuite été évaluées sur huit critères, quatre concernant le rôle de l'université dans la formation dispensée, les quatre autres s'appliquant aux élèves-professeurs, jugés sur leur prestation devant élèves mais aussi sur leurs connaissances théoriques. En septembre, un tableau récapitulatif de ces notes sera publié. Les instituts de formations jugés satisfaisants auront la possibilité de recruter davantage d'étudiants que ceux qui seront jugés médiocres. Les moins bons se verront peut-être retirer leur accréditation, ce qui leur interdira de continuer à fonctionner. Il n'y a peut-être rien à redire à cela, sinon que, lorsque nous nous conformons au programme imposé, comme le font les enseignants tenus d'appliquer les principes de la stratégie nationale pour la lecture, nous devons nous efforcer de ne pas renoncer à nos principes, tout en faisant en sorte que nos collègues conservent leur poste, parce que nous gardons le droit de recruter des étudiants et, en nous assurant que les gens que nous formons trouvent un emploi, parce qu'ils remplissent les critères définis par le diplôme de certification. Par conséquent, il nous reste peu de marge pour tenter des expériences mais les théories sur l'apprentissage de la lecture sont toujours en évolution, à l'état d'ébauche, et nécessitent des révisions constantes à la lumière de nouvelles perspectives conceptuelles et expérimentales, faute de quoi elles ne sont d'aucune utilité.

En tant qu'universités, travaillant avec des enseignants locaux et d'autres collègues de la profession, nous sommes constamment engagés dans des recherches productives qui nourrissent notre enseignement car, pour être efficace, le modèle pédagogique que nous proposons à nos étudiants doit être une synthèse des théories et des pratiques les plus rentables. Nos étudiants, comme les élèves qui pratiquent l'heure de lecture, ont aussi besoin de temps pour "revoir les points importants, se livrer à une réflexion personnelle et consolider les acquis ", mais à la lumière des recherches et des théories récentes et pertinentes, autant que de leurs pratiques de classe. Ils ont besoin de développer une prise de conscience critique de tous les aspects de leur enseignement de la lecture et de l'expression écrite, au lieu d'être confinés par la camisole de force de la stratégie nationale pour la lecture et du Training Curriculum. Car, comme l'affirme la profession de foi de l'université de Brighton :

« La caractéristique distinctive de l'enseignement supérieur (qui est concrétisée par l'obtention d'un diplôme universitaire) est le degré de maîtrise d'un domaine d'études donné et la qualité de l'intelligence critique que cette maitrise suppose. Toute tentative pour réduire le champ des diplômes de l'enseignement supérieur en lui imposant de limiter ses objectifs ou de réduire ses ambitions intellectuelles sera considérée comme un refus de préserver et d'améliorer les exigences de l'université ${ }^{19}$. » 


\section{...Ellis et Tilda sont toujours dans un bateau}

Ellis et Tilda montrent que les enfants apportent avec eux à l'école différents modèles de maîtrise de la lecture et que leurs expériences et leurs attitudes doivent toujours être le point de départ de tout enseignement. Il n'existe pas de définition simple de cette notion, de même qu'il ne peut y avoir une seule façon de l'enseigner. C'est un fait qu'ignorent conjointement le Training Curriculum et le modèle de référence de la stratégie nationale pour la lecture, qui ne proposent de la lecture qu'un modèle fonctionnel. Mais tant que les élèves-professeurs et les enseignants en exercice ne seront pas imprégnés de cette réalité, ils risquent d'interpréter le curriculum comme une liste de compétences à faire acquérir par la pratique d'un ensemble de tâches, plutôt que comme une activité sociale, culturelle et historique complexe qui met en jeu des processus à la fois affectifs et cognitifs. C'est pourquoi nos enfants conserveront leur handicap.

\section{NOTES}

1. DfEE : Department of Education and Employment, le ministère de l'Éducation et de l'Emploi (le terme «emploi» a été ajouté par le nouveau gouvernement travailliste), Standards and Effectiveness Unit, The National Literacy Strategy : Framework for Teaching, London, DfEE, 1998.

2. Les écoles primaires accueillent les enfants de 5 à 11 ans.

3. II s'agit des établissements d'enseignement supérieur, dont un grand nombre possèdent des instituts de pédagogie où les enseignants sont formés à la fois pour le primaire et le secondaire. Les enseignants déjà titulaires d'une licence peuvent préparer en un an le Postgraduate Certificate of Education (PCE) pour obtenir le statut de professeur certifié (Qualified Teacher Status, QTS). Ceux qui ne possèdent pas de diplôme suivent en général une formation de quatre ans pour obtenir leur licence (degree) et leur certification (QTS). Tous les élèves-professeurs doivent effectuer un stage d'une durée substantielle et réglementée dans des établissements scolaires.

4. Office for Standards in Education (OFSTED.

5. J. Bruner, The Process of Education, New-York, Vintage, 1960.

6. DfEE, 1993, § 1.

7. 1988, 10.4 .

8. G Brooks, A. K. Pugh, I. Schagen, Reading Performance at Nine, Slough, National foundation for Educational Research, 1996; Qualifications and Curriculum Authority, «Standards in English at Key Stage 23 - English Maths and Science ", Report on the National Curriculum Assessment for 11 years old, London, Qualifications and Curriculum Authority, 1998.

9. National Literacy Strategy.

10. The National Year of Reading.

11. «Putting the flesh on the bones of the National Curriculum ». (NdlR)

12. DfEE, Initial Teacher Training National Curriculum for Primary English, Annex B of Requirements for Courses of Initial Teacher Training, Circular 10/97, London, DfeEE, section 1, 1997.

13. Le secondaire accueille les élèves de 11 à 16 ans, âge limite de l'obligation scolaire. 
14. Lorraine Dawes, The National Literacy Strategy, a position, article publié par la National Association for Teachers of English (NATE), 1998.

15. DfEE, 1997, § 66.

16. Literary Task Force, The Implentation of the National Literacy Strategy, London, DfEE, 1997, § 64 .

17. DfEE, 1997, p. 45.

18. L. Poulson, D. Wray, J. Medwell, Subject Knowledge and Practice in Primary Literacy Teaching, article présenté à la conférence annuelle de la British Education Research Association, York, 1997.

19. The Directorate, Channel, University de Brighton, publication interne, décembre 1996.

\section{RÉSUMÉS}

Cet aperçu critique des modifications que vont subir les programmes de l'enseignement primaire et la formation des enseignants atteste du désir du gouvernement d'élever le niveau de maîtrise de la lecture et de l'expression écrite. Il explique de quelle façon le professionnalisme et l'autonomie des enseignants de tous niveaux sont, malgré tout, menacés par cette politique éducative.

This critical glimpse into the changes to be brought about in the curriculum in primary schools and in teacher training shows the wishes of the government to raise the level of reading and writing. It explains how the professionalism and the autonomy of teachers at all levels are, nevertheless, threatened by this educational policy.

Este compendio critico de los cambios que conocerán los programas de la enseñanza primaria y la formación de los maestros atestiguan el deseo del gobierno de aumentar el nivel de maestria de la lectura y de la expresión escrita. Explica de qué manera esta política educativa amenaza, a pesar de todo, el profesionalismo y la autonoma de los docentes de todos los niveles.

\section{INDEX}

Mots-clés : enseignement primaire, expression écrite, formation des enseignants, lecture, politique éducative, programme d'enseignement

Index géographique : Angleterre

\section{AUTEURS}

\section{CAROLE KING}

Senior Lecturer, School of Education, University of Brighton, Grande- Bretagne. 